\title{
Will a hiding box provide stress reduction for shelter cats?
}

Universiteit Utrecht

\section{C.M. Vinke ${ }^{\mathbf{1}}$ | L.M. Godijn ${ }^{\mathbf{1}}$ | W.J.R. van der Leij ${ }^{\mathbf{2}}$}

1 - Department of Animals, Science and Society, Faculty of Veterinary Medicine, University of Utrecht, P.O. Box 80166, 3508 TD Utrecht, The Netherlands.
2 - Department of Clinical Sciences of Companion Animals, Shelter Medicine Program,

Faculty of Veterinary Medicine, University of Utrecht, The Netherlands.

\section{I ntroduction}

Domestic cats (Felis sylvestris catus) can experience serious stress in shelters. Stressful experiences can have a major impact on the cats' welfare and may cause higher incidences of infectious diseases in the shelters due to raised cortisol levels causing immunodeficiency. Kry and Casey (2007) found stress reducing effects of hiding boxes on cats in American shelters.

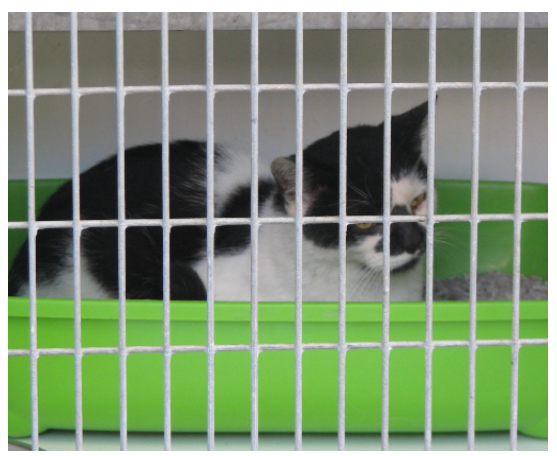

The aim of the present study was to determine the effect of a hiding box on the stress levels of newly arrived cats in a Dutch animal shelter (which considerably differ from American conditions).

\section{Material and methods}

19 newly arrived shelter cats were randomly divided into two groups, with $(\mathrm{N}=10)$ and without a hiding box $(\mathrm{N}=9)$. To determine the stress levels of the research animals, behavioural observations were done during a 14 day period with the help of the CatStress-Score (CSS) as developed by Kessler and Turner (1997).

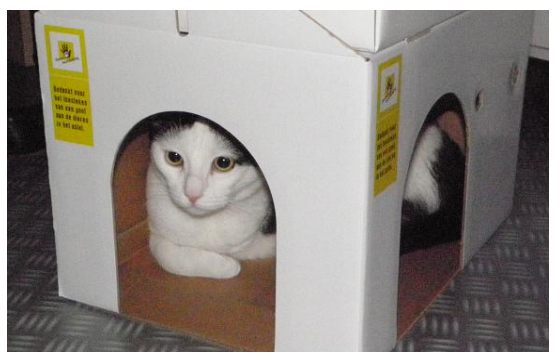

\section{Results}

The main results of this study are, that:

1) a significant different mean CSS was found between the groups on observation day 3 and 4, whereby the hiding box group had a lower mean CSS $(p<0.01)$;

2 ) the mean CSS of the hiding box group showed minimal variance, meaning that the hiding box had its effect on most experimental cats, whereas, high variance could be seen in the group without hiding boxes;

3) the mean CSS for both groups was equal at day 14 , but this level of recovery was already reached around day 3 in the hiding box group.

\section{Discussion}

These findings suggest that cats provided with a hiding box were able to recover faster in their new environment compared to cats without a hiding box, as measured by the CCS. This finding is in line with other studies.

\section{Conclusions}

The findings of the present study showed that the hiding box is an important type of enrichment for the cat to cope effectively with stressors in a shelter environment at least the first weeks after intake. Further research is needed to study the effect of a hiding box on the long term and in cat group housing systems.

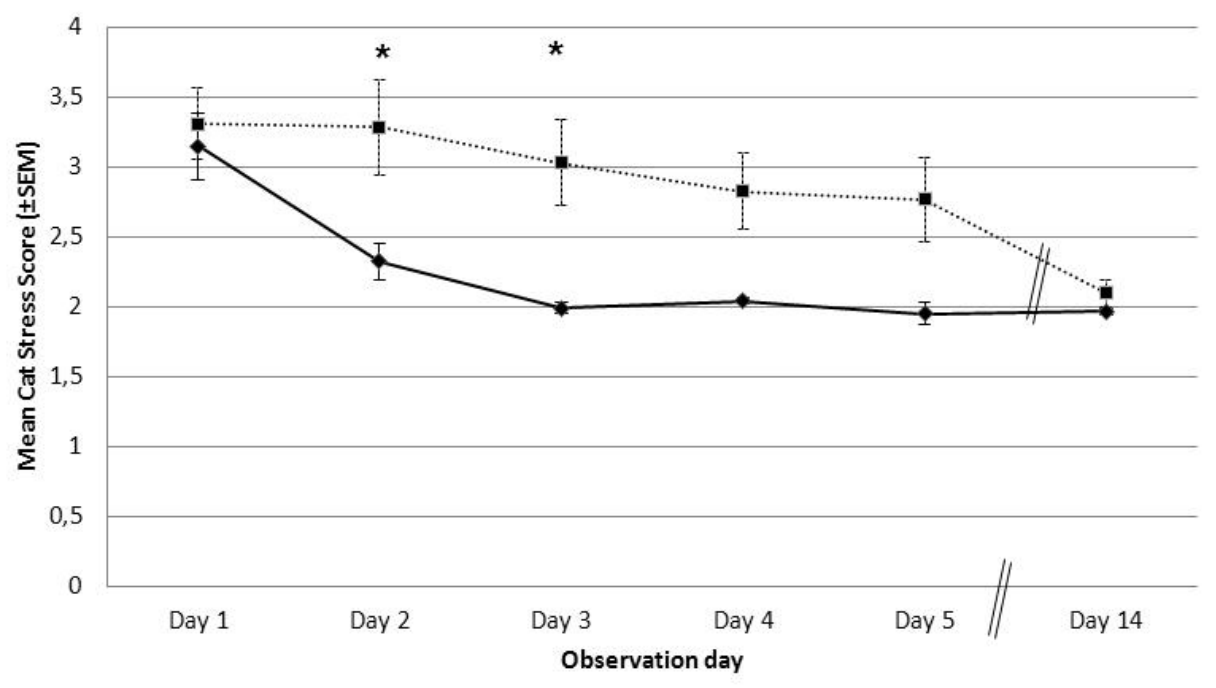

FI GURE 1

Mean Stress Scores ( \pm S.E.M.) of the hiding box group and the control group on each observation day (i.e. day $1-5$ and day 14) ( significant $p<0.01$, Bonferroni corrected).

Hiding box group.

.......... Control group without hiding box.

\section{References}

Kessler, M., Turner, D. 1997.

Stress and adaptation of cats (Felis silvestris catus) housed singly, in pairs and in groups in boarding catteries. Animal Welfare 6(3), 243-254.

Kry, K., Casey, R. 2007.

The effect of hiding enrichment on stress levels and behaviour of Domestic cats (Felis sylvestris catus) in a shelter setting and the implications for adoption potential. Animal Welfare 16(3), 375-383. 